\title{
Identifying Optimal Components in a Reliability System
}

\author{
Josep Freixas and Montserrat Pons
}

\begin{abstract}
The first step in a reliability optimization process is to make a reliability assessment for each component in the system. If this assessment is made in a qualitative way, by grouping together components with the same reliability, and establishing a prevalence order among groups, is there a way to decide which components have the greatest Birnbaum measure without computing the exact value of this measure? In this paper, three relations between components are introduced and studied, and it is proved that they are useful for selecting the components that have the biggest effect on the system reliability in the sense of Birnbaum. An algorithm that uses the results in the paper to select these important components is also provided.
\end{abstract}

Index Terms-Birnbaum's measure of reliability importance, important components, pre-ordering relation.

\section{ACRONYM $^{1}$}

CSS

complete semi-coherent structures

\section{NOTATION}

$N \quad$ the set of components, which are assumed to be numbered consecutively from 1 to $n$.

$x_{i} \quad$ random variable associated to component $i$ : $x_{i}=1$ if component $i$ is functioning, $x_{i}=0$ otherwise. The variables $x_{i}$ are assumed to be $s$-independent.

$\mathbf{x} \quad$ state vector, $\mathbf{x}=\left(x_{1}, x_{2}, \ldots, x_{n}\right)$.

$\left(a_{i}, \mathbf{x}\right) \quad$ it represents the state vector $\mathbf{x}$ where the $i$ th component has been changed to $a$.

$\phi(\mathbf{x}) \quad$ structure (Boolean) function of the system: $\phi(\mathbf{x})=1$ if system is functioning, $\phi(\mathbf{x})=0$ otherwise.

$(N, \phi) \quad$ semicoherent system, its structure function $\phi$ is nondecreasing in each argument, $\phi(\mathbf{0})=0$, and $\phi(\mathbf{1})=1$.

$p_{i} \quad$ reliability of the $i$ th component, $\operatorname{Pr}\left(x_{i}=1\right)$.

p reliability vector, $\left(p_{1}, p_{2}, \ldots, p_{n}\right)$.

$\left(a_{i}, \mathbf{p}\right) \quad$ reliability vector $\mathbf{p}$ with the $i$ th component of $\mathbf{p}$ changed to $a$.

Manuscript received May 8, 2006; revised May 11, 2007; accepted June 25, 2007. This work was supported in part by Grant MTM 2006-06064 of "Ministerio Español de Educación y Ciencia y el Fondo Europeo de Desarrollo Regional," and by Grant SGRC 2005-00651 of "Generalitat de Catalunya." Associate Editor: G. Levitin.

The authors are with the Department of Applied Mathematics 3, School of Engineering of Manresa, Technical University of Catalonia, Spain (e-mail: josep. freixas@upc.edu; montserrat.pons@upc.edu).

Digital Object Identifier 10.1109/TR.2008.916888

${ }^{1}$ The singular and plural of an acronym are always spelled the same.
$(N, \pi) \quad$ semicoherent structure defined by the set $\pi$ of path sets in $N$.

$\left(N, \pi^{m}\right) \quad$ semicoherent structure defined by the set $\pi^{m}$ of minimal path sets in $N$.

$h(\mathbf{p}) \quad$ system reliability, $h(\mathbf{p})=E(\phi(\mathbf{x}))$; $h(\mathbf{p})=\sum_{S \in \pi} \prod_{i \in S} p_{i} \prod_{i \in N \backslash S}\left(1-p_{i}\right)$.

$I_{i}^{B}(\mathbf{p}) \quad$ Birnbaum's measure of reliability importance of component $i$ : the partial derivative of $h$ with respect to $p_{i}$, i.e., $\partial h / \partial p_{i}$, or $h\left(1_{i}, \mathbf{p}\right)-h\left(0_{i}, \mathbf{p}\right)$.

\section{INTRODUCTION}

$\mathbf{E}$ NGINEERS are often faced with the task of identifying the components of a system that have the biggest effect on the system reliability. These components deserve especial interest in many circumstances (if the reliability of the system has to be increased, if certain types of improvement in input parameters has to be done, etc.). In general, the identification of these components is done by using some measure of component importance.

One of the most used measures for determining the importance of a component in a binary semicoherent system is Birnbaum's measure of reliability importance [1], which is mainly used in classical sensitivity analysis. The calculation of the Birnbaum measure can be difficult if the number of components in the system is not small, but it is impossible to find its exact value if component reliabilities are not precisely known.

The aim of this paper is to investigate the problem of finding the component(s) that have the biggest effect on the system reliability in the sense of the Birnbaum measure, i.e., the components with the highest Birnbaum measure, when only the structure function of the system, and the ranks of components' reliabilities (but possibly not their exact values) are known. We refer to this (these) component(s) as the most important component(s) in the sense of Birnbaum in a reliability system. To help identify these (most important) components, we use some binary relations defined on the set of nodes of the system, i.e., binary relations that do not depend on the nature of the components, but only depend on the structure of the system.

Some work has previously been done in similar contexts. In particular, the binary relation called the "criticality relation" was introduced in [2] as a tool to find an optimal component arrangement that maximizes system reliability, and some other relations between nodes have also been considered in the literature [3], [4]. These relations have subsequently been used to compare the Birnbaum measure between system components [5]-[7]. 
TABLE I

THE MOST IMPORTANT COMPONENTS IN A 2-OUT-OF-3 SYSTEM

\begin{tabular}{ccc} 
Components' reliabilities & $I^{B}$ measure & Most important \\
\hline$p_{3} \geq p_{2} \geq p_{1} \geq \frac{1}{2}$ & $I_{3}^{B} \geq I_{2}^{B} \geq I_{1}^{B}$ & component 3 \\
$p_{3} \geq p_{2} \geq \frac{1}{2}>p_{1}$ & $I_{2}^{B} \geq I_{3}^{B} \geq I_{1}^{B}$ & component 2 \\
$p_{3} \geq \frac{1}{2} \geq p_{2} \geq p_{1}$ & $I_{2}^{B} \geq I_{1}^{B} \geq I_{3}^{B}$ & component 2 \\
$\frac{1}{2} \geq p_{3} \geq p_{2} \geq p_{1}$ & $I_{1}^{B} \geq I_{2}^{B} \geq I_{3}^{B}$ & component 1 \\
\hline
\end{tabular}

In this paper, two new binary relations (pre-orderings) between nodes are introduced, and it is proved that they are stronger than the criticality relation, i.e., they imply it. The main results of this paper show that these newly defined binary relations between components induce a ranking between their Birnbaum measures, and so they play a key role (together with the criticality relation) in identifying the most important component(s) in the sense of Birnbaum in a complex reliability system. Using these three pre-orderings, we are able to construct an algorithm that selects a set of components, which we call potentially important, and which contain the most important one(s).

Similar work could be done using other alternative importance measures. In [8], and [9] the criticality relation is used to study the importance of components in the sense of FussellVesely measure. The study we propose in this paper might also be adapted for other reliability importance measures, such as those considered for example in [10], or [11].

Recall that $k$-out-of- $n$ systems are functioning iff at least $k$ of the $n$ components are functioning, i.e., $\phi(\mathbf{x})=1$ if $\sum_{i=1}^{n} x_{i} \geq$ $k$, and $\phi(\mathbf{x})=0$ otherwise. Series, and parallel systems can be considered particular cases for $k=n$, and $k=1$ respectively.

Series, and parallel systems are conspicuous examples of systems wherein the ordering between components' reliabilities determines the most important component(s). Indeed, the most important component in a series system is the least reliable one, while in a parallel system the most important component is the most reliable one.

On the contrary, in a 2-out-of-3 system, the simplest intermediate case between parallel and series systems, one can easily check that the election of the most important component not only depends on the ordering between components' reliabilities, but it also depends on the actual values of these reliabilities. The following table summarizes all the possible situations, having in mind that all components play interchangeable roles.

The first line in Table I is a particular example of a result in [12] where it is proved that, in a $k$-out-of- $n$ system with independent components, Birnbaum's importance ordering between components agrees with the ordering between their corresponding reliabilities iff all of them are greater than $(k-1) /(n-$ $1)$.

In general, as asserted in [13], by examining Birnbaum's importance measures of components in a given system, system analysts could gain better insight into the system design without resorting to mathematical programming methods as described in [14], or [15].

To end this introduction, we summarize in the following lemma some basic properties of the Birnbaum measure $I^{B}(\mathbf{p})$. The proof is omitted because parts (i)-(iii) are trivial, (iv) is a consequence of (i) \& (iii), and (v) follows from the law of total probability which leads to the following expressions (for $a=0,1)$ :

$$
\begin{aligned}
& h\left(a_{i}, \mathbf{p}\right)=p_{j} h\left(a_{i}, 1_{j}, \mathbf{p}\right)+\left(1-p_{j}\right) h\left(a_{i}, 0_{j}, \mathbf{p}\right) \\
& h\left(a_{j}, \mathbf{p}\right)=p_{i} h\left(1_{i}, a_{j}, \mathbf{p}\right)+\left(1-p_{i}\right) h\left(0_{i}, a_{j}, \mathbf{p}\right) .
\end{aligned}
$$

Recall that a component in a system is irrelevant if its failure does not affect the performance of the system, and it is relevant otherwise. A semicoherent system without irrelevant components is said to be coherent.

Lemma 1.1: Let $(N, \pi)$ be a semicoherent structure, $\mathbf{p}, \mathbf{p}^{*} \in$ $[0,1]^{n}, i \in N$. Then

(i) $0 \leq I_{i}^{B}(\mathbf{p}) \leq 1$.

(ii) If $i$ is an irrelevant component, then $I_{i}^{B}(\mathbf{p})=0$.

(iii) If $i$ is a relevant component, and $\mathbf{p} \in(0,1)^{n}$, then $I_{i}^{B}(\mathbf{p})>0$.

(iv) If $p_{i}<p_{i}^{*}$, and $p_{k}=p_{k}^{*}$ for all $k \neq i$, then $h(\mathbf{p}) \leq h\left(\mathbf{p}^{*}\right)$. Moreover, if $i$ is relevant, and $\mathbf{p} \in(0,1)^{n}$, then we get $h(\mathbf{p})<h\left(\mathbf{p}^{*}\right)$.

(v) For all $j \in N, j \neq i$, we have

$$
\begin{aligned}
I_{i}^{B}(\mathbf{p})-I_{j}^{B}(\mathbf{p})= & \left(p_{j}-p_{i}\right)\left[h\left(1_{i}, 1_{j}, \mathbf{p}\right)+h\left(0_{i}, 0_{j}, \mathbf{p}\right)\right. \\
& \left.-h\left(1_{i}, 0_{j}, \mathbf{p}\right)-h\left(0_{i}, 1_{j}, \mathbf{p}\right)\right] \\
& +\left[h\left(1_{i}, 0_{j}, \mathbf{p}\right)-h\left(0_{i}, 1_{j}, \mathbf{p}\right)\right] .
\end{aligned}
$$

The organization of the paper is as follows. In Section II, three pre-orderings are considered on the set of components, and their properties are established. In Section III, three main theorems are stated which relate Birnbaum's measures of two different components linked by some of the previously defined pre-orderings. Section IV contains the conclusions. An algorithm that uses the theorems of Section III is shown in the first part of the Appendix. The second part of it is devoted to the proofs of the main statements of the paper.

\section{SOME USEFUl PRE-ORdERINGS ON $N$}

We are going to introduce three binary relations between components that will be helpful to solve the proposed problem. One of them is already well known, and it is a particular pre-ordering between components. It is known as the desirability relation in game theory (see, e.g., [16], and [17]), or as the criticality relation in reliability theory (see [2]), and it is a useful tool to compare the relative strength of the components of a system. A classification theorem (see [18]) allows us to generate and count, up to isomorphism, the number of semi-coherent structures for which the criticality relation is complete.

The other two pre-orderings are new. Both of them extend to (imply) the criticality relation, and the simultaneous verification of them provides us with a new characterization of the irrelevance.

Definition 2.1: Let $(N, \pi)$ be a semicoherent structure. Given two elements $i, j \in N$, we consider the following binary relations.

(i) The external domination relation. $i \vDash j$ iff $i=j$, or $[S \in \pi, j \in S, i \notin S \Rightarrow S \backslash\{j\} \in \pi]$.If $i \vDash j$, we say that component $i$ externally dominates $j$. 


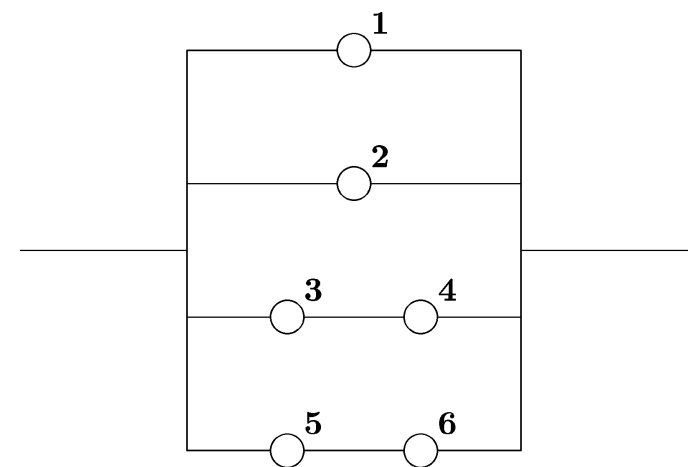

Fig. 1. Coherent structure with $N=\{1,2,3,4,5,6\}$, and $\pi^{m}=$ $\{\{1\},\{2\},\{3,4\},\{5,6\}\}$.

(ii) The internal domination relation. $i \unrhd j$ iff $i=j$, or $[S \in \pi, i, j \in S \Rightarrow S \backslash\{j\} \in \pi]$.If $i \unrhd j$, we say that component $i$ internally dominates $j$.

(iii) The criticality relation. $i \succeq j$ iff $[S \cup\{j\} \in \pi \Rightarrow$ $S \cup\{i\} \in \pi$, whenever $S \subseteq N \backslash\{i, j\}]$. If $i \succeq j$ we say that component $i$ is at least as critical as component $j$.

Proposition 2.2: Let $(N, \pi)$ be a semicoherent structure. The external domination relation, the internal domination relation, and the criticality relation are transitive relations on $N$.

It is clear that the three considered binary relations are reflexive. The former proposition shows that they are pre-orderings, i.e., they are reflexive and transitive. It is important to note that these pre-orderings can be characterized using minimal path sets. This fact considerably reduces the computational work for large systems.

Proposition 2.3: Let $(N, \pi)$ be a semi-coherent structure, and $i, j$ be different elements in $N$.

(i) $i \vDash j$ iff $\left[S \in \pi^{m}, j \in S \Rightarrow i \in S\right]$

(ii) $i \unrhd j$ iff $\left[S \in \pi^{m}, j \in S \Rightarrow(S \cup\{i\}) \backslash\{j\} \in \pi\right]$

(iii) $i \succeq j$ iff $\left[S \in \pi^{m}, j \in S, i \notin S \Rightarrow(S \cup\{i\}) \backslash\{j\} \in \pi\right]$

The following example shows that in general the three pre-orderings are not antisymmetric (i.e., they are not ordering relations), and also that they are not total (i.e., there exist incomparable elements).

Example 2.4: Let $(N, \pi)$ be a coherent structure such that $N=\{1,2,3,4,5,6\}$, and $\pi^{m}=\{\{1\},\{2\},\{3,4\},\{5,6\}\}$ (Fig. 1). Then

(i) $3 \vDash 4$, and $4 \vDash 3 ; 5 \vDash 6$, and $6 \vDash 5$; and there are no other links by the external domination relation.

(ii) $1 \unrhd 2$, and $2 \unrhd 1 ; 1,2 \unrhd 3,4,5,6$; and there are no other links by the internal domination relation.

(iii) $1 \succeq 2$, and $2 \succeq 1 ; 3 \succeq 4$, and $4 \succeq 3 ; 5 \succeq 6$, and $6 \succeq 5 ; 1,2 \succeq 3,4,5,6$; and there are no other links by the criticality relation.

Definition 2.5: Let $(N, \pi)$ be a semicoherent structure. Given two elements $i, j \in N$, the following binary relations will be used:

(1.a) $i \vdash j$ iff $i \vDash j$, and $j \not \models i$

(1.b) $i \boxminus j$ iff $i \vDash j$, and $j \vDash i$

(2.a) $i \triangleright j$ iff $i \unrhd j$, and $j$ 电 $i$

(2.b) $i \bowtie j$ iff $i \unrhd j$, and $j \unrhd i$

(3.a) $i \succ j$ iff $i \succeq j$, and $j \nsucceq i$

(3.b) $i \equiv j$ iff $i \succeq j$, and $j \succeq i$
Obviously, due to the reflexivity of the three pre-orderings, $\succeq, \vDash, \unrhd$, we have

$$
i=j \Rightarrow i \equiv j, i \text { \# } j \text {, and } i \bowtie j ;
$$

but the converse implications do not hold because the pre-orderings are not antisymmetric.

These binary relations are not independent. The following proposition states some relations among them.

Proposition 2.6: Let $(N, \pi)$ be a semicoherent structure, and $i, j$ be different elements in $N$.

(i) $i \vDash j \Rightarrow i \succeq j$

(ii) $i \vdash j \Rightarrow i \succ j$

(iii) $i \unrhd j \Rightarrow i \succeq j$

(iv) $i \triangleright j \Rightarrow i \succ j$.

The former proposition proves that each one of the new preorderings implies the criticality relation. Moreover, because Example 2.4 has shown that neither the converse of (i) nor the converse of (iii) holds, then we conclude that the two new pre-orderings are stronger than the criticality relation. However, notice that if $i, j$ are different components in $N$, and there is no $S \in \pi$ with $i, j \in S$, then $i \unrhd j$ iff $i \succeq j$.

The following proposition provides a new characterization of irrelevant components using external, and internal subordination pre-orderings.

Proposition 2.7: Let $(N, \pi)$ be a semicoherent structure, and $i, j$ be different elements in $N$. Then

$i \vDash j$, and $i \unrhd j \Leftrightarrow j$ is an irrelevant component.

\section{Method to Find the Most IMPortant Components}

Recall that our goal is to select the most important components in a reliability system $((N, \pi), \mathbf{p})$ where $\mathbf{p}$ is a reliability vector with a known ordering between its components. Our way of doing this is to construct a set of potentially important components, by discarding, from the whole set of components, those that are strictly less important than another one in the set.

The theorems in this section explain which one of two components has maximum $I^{B}$-measure whenever they are comparable by some of the foregoing pre-orderings. In this case, the component with minimum measure is clearly not the most important, and so it can be removed from the set of potentially important components. This principle is the base for the construction of a step-by-step algorithm for finding a set of potentially important components for all semicoherent structures $(N, \pi)$, and for all reliability vectors $\mathbf{p}$ in $[0,1]^{n}$, using Theorems $3.3,3.4$, and 3.5.

Because irrelevant components have $I^{B}$-measure zero for all reliability vectors $\mathbf{p}$ (Lemma 1.1-(ii)), they will never be the most important components in a system. This is why, in the rest of this paper, we assume that all components are relevant.

We start the section with a proposition that shows the utility of the former binary relations to solve the problem of finding the component with the biggest effect on the system reliability.

Proposition 3.1: Let $(N, \pi)$ be a semicoherent structure, $\mathbf{p} \in$ $[0,1]^{n}$, and $i, j$ be different elements in $N$.

(i) $i \vDash j \Rightarrow h\left(0_{i}, 0_{j}, \mathbf{p}\right)=h\left(0_{i}, 1_{j}, \mathbf{p}\right)$. If $\mathbf{p} \in(0,1)^{n}$ then the converse is also true.

(ii) $i \unrhd j \Rightarrow h\left(1_{i}, 0_{j}, \mathbf{p}\right)=h\left(1_{i}, 1_{j}, \mathbf{p}\right)$. If $\mathbf{p} \in(0,1)^{n}$ then the converse is also true. 
(iii) $i \succeq j \Rightarrow h\left(1_{i}, 0_{j}, \mathbf{p}\right)-h\left(0_{i}, 1_{j}, \mathbf{p}\right) \geq 0$.

(iv) $i \succ j$, and $\mathbf{p} \in(0,1)^{n} \Rightarrow h\left(1_{i}, 0_{j}, \mathbf{p}\right)-h\left(0_{i}, 1_{j}, \mathbf{p}\right)>0$.

The following proposition proves that, if two components are not conveniently related, then there are no arguments to discard any of them as potentially important components in the system.

Proposition 3.2: Let $(N, \pi)$ be a semicoherent structure, and let $i, j$ be different elements in $N$. Also let $x, y \in[0,1]$. Then

(i) If $i \succeq j$, and $i \not \models j$, then there exists a reliability vector $\mathbf{p} \in[0,1]^{n}$ such that $p_{i}=x, p_{j}=y$, and $I_{i}^{B}(\mathbf{p})-$ $I_{j}^{B}(\mathbf{p})=x-y$.

(ii) If $i \succeq j$, and $i \not j$, then there exists a reliability vector $\mathbf{p} \in[0,1]^{n}$ such that $p_{i}=x, p_{j}=y$, and $I_{i}^{B}(\mathbf{p})-$ $I_{j}^{B}(\mathbf{p})=y-x$.

(iii) If $i \nsucceq j$, then there exists a reliability vector $\mathbf{p} \in[0,1]^{n}$ such that $p_{i}=x, p_{j}=y$, and $I_{j}^{B}(\mathbf{p})-I_{i}^{B}(\mathbf{p})=1$.

The following theorem proves that if a component $i$ externally dominates another component $j$, and its reliability $p_{i}$ is lower than $p_{j}$, then component $j$ can be discarded as potentially important. But if $p_{i}$ is greater than $p_{j}$, then neither of the two components can be discarded, i.e., both of them could still be the most important component in the system.

Theorem 3.3: (On the external domination relation) Let $(N, \pi)$ be a semicoherent structure, $\mathbf{p} \in[0,1]^{n}$, and $i, j$ be different relevant components in $N$. Then

(i) $i \vDash j$, and $p_{i} \leq p_{j} \Rightarrow I_{i}^{B}(\mathbf{p}) \geq I_{j}^{B}(\mathbf{p})$.

(ii) $i \vDash j$, and $\mathbf{p} \in(0,1)^{n}$ with $p_{i}<p_{j} \Rightarrow I_{i}^{B}(\mathbf{p})<I_{j}^{B}(\mathbf{p})$.

(iii) if $i \vdash j$, and $p_{i}>p_{j}$, then there exist vectors $\mathbf{p}^{*}, \mathbf{p}^{\diamond} \in$ $[0,1]^{n}$ with $p_{i}^{*}=p_{i}^{\diamond}=p_{i}$, and $p_{j}^{*}=p_{j}^{\diamond}=p_{j}$, such that $I_{i}^{B}\left(\mathbf{p}^{*}\right)<I_{j}^{B}\left(\mathbf{p}^{*}\right)$, and $I_{i}^{B}\left(\mathbf{p}^{\diamond}\right)>I_{j}^{B}\left(\mathbf{p}^{\diamond}\right)$.

In the next theorem, it is proved that if a component $i$ internally dominates another component $j$, and its reliability $p_{i}$ is greater than $p_{j}$, then component $j$ can be discarded as potentially important. But if $p_{i}$ is lower than $p_{j}$, then neither of the two components can be discarded.

Theorem 3.4: (On the internal domination relation) Let $(N, \pi)$ be a semicoherent structure, $\mathbf{p} \in[0,1]^{n}$, and $i, j$ be different relevant components in $N$. Then

(i) $i \unrhd j$, and $p_{i} \geq p_{j} \Rightarrow I_{i}^{B}(\mathbf{p}) \geq I_{j}^{B}(\mathbf{p})$.

(ii) $i \unrhd j$, and $\mathbf{p} \in(0,1)^{n}$ with $p_{i}>p_{j} \Rightarrow I_{i}^{B}(\mathbf{p})>I_{j}^{B}(\mathbf{p})$.

(iii) if $i \triangleright j$, and $p_{i}<p_{j}$, then there exist vectors $\mathbf{p}^{*}, \mathbf{p}^{\diamond} \in$ $[0,1]^{n}$ with $p_{i}^{*}=p_{i}^{\diamond}=p_{i}$, and $p_{j}^{*}=p_{j}^{\diamond}=p_{j}$, such that $I_{i}^{B}\left(\mathbf{p}^{*}\right)<I_{j}^{B}\left(\mathbf{p}^{*}\right)$, and $I_{i}^{B}\left(\mathbf{p}^{\diamond}\right)>I_{j}^{B}\left(\mathbf{p}^{\diamond}\right)$

The last theorem proves that if a component $i$ is more critical than another component $j$, and their reliabilities coincide, then component $j$ can be discarded as potentially important. But if $p_{i}$ is different of $p_{j}$, then none of the two components can be discarded except in the cases provided by the former two theorems.

Theorem 3.5: (On the criticality relation) Let $(N, \pi)$ be a semicoherent structure, $\mathbf{p} \in[0,1]^{n}$, and $i, j$ be different relevant elements in $N$. Then,

(i) $i \succeq j$, and $p_{i}=p_{j} \Rightarrow I_{i}^{B}(\mathbf{p}) \geq I_{j}^{B}(\mathbf{p})$.

(ii) $i \succ j$, and $\mathbf{p} \in(0,1)^{n}$ with $p_{i}=p_{j} \Rightarrow I_{i}^{B}(\mathbf{p})>I_{j}^{B}(\mathbf{p})$.

(iii) if $i \succ j, i \nvdash j, i \not j$, and $p_{i} \neq p_{j}$, then there exist vectors $\mathbf{p}^{*}, \mathbf{p}^{\diamond} \in[0,1]^{n}$ with $p_{i}^{*}=p_{i}^{\diamond}=p_{i}$, and $p_{j}^{*}=p_{j}^{\diamond}=p_{j}$, such that $I_{i}^{B}\left(\mathbf{p}^{*}\right)<I_{j}^{B}\left(\mathbf{p}^{*}\right)$, and $I_{i}^{B}\left(\mathbf{p}^{\diamond}\right)>I_{j}^{B}\left(\mathbf{p}^{\diamond}\right)$

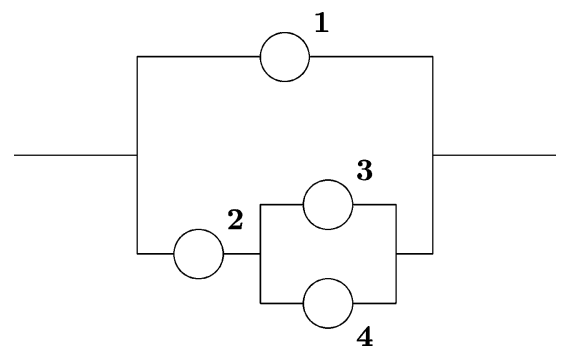

Fig. 2. Coherent structure with $N=\{1,2,3,4\}$, and $\pi^{m}=$ $\{\{1\},\{2,3\},\{2,4\}\}$.

The theorems in this section allow us to know, for all possible ways in which two components are related by any of the pre-orderings considered, and for all orderings between their reliabilities, which one of these situations happens: $a$ ) one of them can be removed from the set of potentially important components, or b) both of them are candidates to be the most important component(s). As a consequence, we can use these theorems to select a list of potentially important components, given any particular ordering of their reliabilities, with ties allowed. Let us remark that the algorithm can be easily implemented (this is done in the Appendix) in a computer whenever the set of minimal path sets is not too large.

We conclude the paper with an example that will help the reader to better understand the provided results.

Example 3.6: Let $\left(N, \pi^{m}\right)$ be a coherent structure such that $N=\{1,2,3,4\}$, and $\pi^{m}=\{\{1\},\{2,3\},\{2,4\}\}$ (Fig. 2). Assume that the ordering between components' reliabilities is $0<p_{2}<p_{1} \leq p_{4} \leq p_{3}<1$. Which is the most important component(s), i.e., which component(s) has the highest Birnbaum measure?

Using Proposition 2.3-(i),(ii) we have

$$
\begin{gathered}
1 \triangleright 2 ; 1 \triangleright 3 \bowtie 4 \\
2 \vdash 3 ; 2 \vdash 4 .
\end{gathered}
$$

Consequently, from Proposition 2.6-(ii),(iii),(iv) we get

$$
1 \succ 2 \succ 3 \equiv 4 .
$$

Now, because $2 \vdash 4$, and $p_{2}<p_{4}$, then we get $I_{2}^{B}(\mathbf{p})>I_{4}^{B}(\mathbf{p})$ using Theorem 3.3, and so component 4 is clearly not the most important one. Using the same argument, component 3 can be discarded. Finally, because $1 \triangleright 2$, and $p_{1}>p_{2}$, then $I_{1}^{B}(\mathbf{p})>$ $I_{2}^{B}(\mathbf{p})$ using Theorem 3.4. Thus component 2 can not be the most important either. As a conclusion, the set of potentially important components reduces to a singleton, component 1 . This tells us that component 1 is the most important in this reliability system.

The use of Theorems 3.3, 3.4, and 3.5 does not always allow us to find directly the most important component as in this example, but this procedure will always allow us to select a set of potentially important components containing the most important component(s).

\section{CONCLUSIONS}

The paper contains a new algebraic method for determining the most important components, in the sense of the Birnbaum 
measure, in a semicoherent system with statistically independent random Boolean variables.

For this purpose, two new pre-ordering relations between the components of the reliability system are introduced (external, and internal domination). These two pre-orderings, as well as the well known criticality pre-ordering (which extends each one of them), are defined through the set $\pi$ of path sets of the system. The three relations are studied in detail, from the algebraic point of view, and are used to derive the main results of the paper, three theorems which respectively correspond to each one of the three pre-ordering relations. These theorems provide us with sufficient conditions for assuring certain inequalities between the Birnbaum measure of reliability importance of two given components. More precisely, the theorems state that if two fixed components $i, j$ are comparable by one of these pre-orderings, and if their reliabilities $p_{i}, p_{j}$ satisfy the corresponding (in)equality, then we can determine a priori the ordering between their Birnbaum's measures $I_{i}^{B}(\mathbf{p}), I_{j}^{B}(\mathbf{p})$, without computing them.

The theorems in this paper extend the results obtained in [5] by using the criticality relation. There it is proved that if component $i$ is strictly greater than $j$ by the criticality relation, and their reliabilities coincide, i.e., $p_{i}=p_{j}$, then the Birnbaum measure of $i$ is strictly greater than that of $j$. In this paper, we prove that if $p_{i} \neq p_{j}$ it is also possible to know which of them has a greater Birnbaum measure, by considering two newly defined stronger pre-orderings on the set of nodes. It is proved that if $i$ is greater than $j$ by the external domination relation, and $p_{i}<p_{j}$, then the Birnbaum measure of $i$ is strictly greater than that of $j$. We come to the same conclusion if component $i$ is greater than $j$ by the internal domination relation, and $p_{i}>p_{j}$. Using these results, we construct a set of potentially important components, i.e., components that may be the most important ones, by discarding from the whole set of components those that are strictly less important than another one.

The results for the criticality relation, and for the two domination relations introduced in this paper, complement each other. In fact, these domination relations are stronger than the criticality relation so that the criticality relation between two components appears more frequently than each one of the two domination relations. On the other hand, the condition $p_{i}=p_{j}$ required to use the criticality relation for discarding a component (Theorem 3.5) is stronger than the inequalities needed to use the domination relations (Theorems 3.3 and 3.4).

If at least two components in a system are related by the criticality relation, our procedure can be applied to find a set of potentially important components. The widest applicability appears when the criticality relation is total (complete semi-coherent structures). The number of complete semi-coherent structures (CSS) of less than 9 components (obtained by programming the four conditions stated in [18] to classify them) is given in Table II, and illustrates that the study presented in this paper has a wide applicability.

It would be interesting to study how the theory introduced in this paper for Birnbaum's measure can be extended to other reliability importance measures such as improvement potential, risk achievement worth, risk reduction worth, or Fussell-Vesely's measure.
TABLE II

NUMBER OF CSS WITH $n$ COMPONENTS

\begin{tabular}{llllrrrrr}
$n$ & 1 & 2 & 3 & 4 & 5 & 6 & 7 & 8 \\
\hline CSS & 1 & 3 & 8 & 25 & 117 & 1171 & 44313 & 16175188
\end{tabular}

Another extension for our work should include multistate monotone systems where components and system are allowed to have an arbitrary (finite) number of states/levels.

Another interesting problem would be to find a subclass of semicoherent structures as wide as possible for which the respective pre-orderings induced for the two most well known measures of structural importance given by Barlow and Proschan, and Birnbaum coincide; i.e., to study the ordinal equivalence of the two structural importance measures.

\section{APPENDIX I \\ ALGORITHM}

Given a semicoherent structure $\left(N, \pi^{m}\right)$, and a particular ordering between the components of the reliability vector $\mathbf{p} \in$ $(0,1)^{n}$, with ties allowed, the following algorithm selects a set of potentially important components using Theorems 3.3, 3.4, and 3.5. It uses three auxiliary procedures: $E D(i, j), I D(i, j)$, and $C R(i, j)$, which are described after the main algorithm, that check respectively if $i \vDash j, i \unrhd j$, and $i \succeq j$.

\section{Main alogoritm}

\section{INPUT:}

- The number $n$ of components.

- The set $\pi^{m}=\left\{S_{1}^{1}, \ldots, S_{t_{1}}^{1}, S_{1}^{2}, \ldots, S_{t_{2}}^{2}, \ldots, S_{1}^{q}, \ldots, S_{t_{q}}^{q}\right\}$ of minimal path sets, where the superindex indicates the number of components in the set, and for each $h(1 \leq h \leq q), t_{h}$ is the number of minimal path sets with $h$ elements. We assume that for all $h(1 \leq h \leq q)$, the sets $S_{1}^{h}, \ldots, S_{t_{h}}^{h}$ are arranged in lexicographic order of their components.

- A partition $N=\bigcup_{k=1}^{r} N_{k}$ of the set $N$ of components $(1 \leq r \leq n)$. We assume that all components in $N_{k}(1 \leq k \leq r)$ have the same reliability $p^{k}$, and that $p^{1}>p^{\overline{2}}>\cdots>p^{r}$.

OUTPUT: The set PIC of potentially important components.

STEP 1 (discarding irrelevant components): For every $i \in N$, for every $h(1 \leq h \leq q)$, and for every $d\left(1 \leq d \leq t_{h}\right)$, check if $i \in S_{d}^{h}$. If $i$ does not belong to any $S_{d}^{h}$, then let $N_{k}=N_{k} \backslash\{i\}$ for the unique value of $k(1 \leq k \leq r)$ such that $i \in N_{k}$, i.e., $p_{i}=p^{k}$, and let $N=N \backslash\{i\}$.

STEP 2 (discarding components within those that have the same reliability): For every $N_{k}(1 \leq k \leq r)$, and for all pair $(i, j) \in N_{k} \times N_{k}$ with $i \neq j$, if $C R(i, j)=1$, and $C R(j, i)=0$, then let $j \in D i s$. In this way, a set Dis is being constructed of elements that will be discarded. We don't discard them yet because they still could help other components to be discarded in the next step. 
STEP 3 (discarding components within those that have different reliability): For every $N_{k}(1 \leq k \leq r)$, for every $N_{l}(k+1 \leq l \leq r)$, and for every $(i, j) \in N_{k} \times N_{l}$, check if $i \unrhd j$, and if $j \vDash i$. If $i \unrhd j$, i.e., $I D(i, j)=1$, then let $j \in D i s$. If $j \vDash i$, i.e. $E D(j, i)=1$, then let $i \in D i s$.

STEP 4: The set of potentially important components is $P I C=N \backslash$ Dis.

\section{Procedure ED}

INPUT: Two different components $i$ and $j$ of $N$.

OUTPUT: The Boolean value $E D(i, j)$, which is 1 if $i \vDash j$, and 0 otherwise.

STEP 0: Initialize $I=1$.

STEP 1: For every $h(1 \leq h \leq q)$, and for every $d\left(1 \leq d \leq t_{h}\right)$, check if $\bar{j} \in S_{d}^{h}$. If this is the case, then if $i \notin S_{d}^{h}$, let $I=0$, and finish step 1 .

STEP 2: Let $E D(i, j)=I$.

\section{Procedure ID}

INPUT: Two different components $i$ and $j$ of $N$.

OUTPUT: The Boolean value $I D(i, j)$, which is 1 if $i \unrhd j$, and 0 otherwise.

STEP 0: Initialize $I=1$.

STEP 1: For every $h(1 \leq h \leq q)$, and for every $d\left(1 \leq d \leq t_{h}\right)$, check if $j \in \overline{S_{d}^{h}}$. If $j \in S_{d}^{h}$, and it is also $i \in S_{d}^{h}$, then let $I=0$, and finish step 1 . If $j \in S_{d}^{h}$ but $i \notin S_{d}^{h}$, then let $T=\left(S_{d}^{h} \cup\{i\}\right) \backslash\{j\}$, and search if there is some $a(1 \leq a \leq h)$ and some $b\left(1 \leq b \leq t_{a}\right)$ such that $T \supseteq S_{b}^{a}$. If no such $S_{b}^{a}$ can be found, then let $I=0$ and finish step 1 .

STEP 2: Let $I D(i, j)=I$.

\section{Procedure CR}

INPUT: Two different components $i$ and $j$ of $N$.

OUTPUT: The Boolean value $C R(i, j)$, which is 1 if $i \succeq j$, and 0 otherwise.

STEP 0: Initialize $I=1$.

STEP 1: For every $h(1 \leq h \leq q)$, and for every $d\left(1 \leq d \leq t_{h}\right)$, check if $j \in S_{d}^{h}$. If $j \in S_{d}^{h}$, and also $i \notin S_{d}^{h}$, then let $T=\left(S_{d}^{h} \cup\{i\}\right) \backslash\{j\}$, and search if there is some $a(1 \leq a \leq h)$ and some $b\left(1 \leq b \leq t_{a}\right)$ such that $T \supseteq S_{b}^{a}$. If no such $S_{b}^{a}$ can be found, then let $I=0$, and finish step 1 .

STEP 2: Let $C R(i, j)=I$

\section{APPENDIX II}

\section{PROOFS}

\section{A. Proof of Proposition 2.2}

To prove that the external domination relation is transitive, assume that $i \vDash j \vDash k$, and that $i \neq j \neq k \neq i$. Suppose that $S \in \pi$ is such that $k \in S$, and $i \notin S$. We must prove that $S \backslash\{k\} \in \pi$. There are two possibilities:

If $j \notin S$, because $j \vDash k$, then $S \backslash\{k\} \in \pi$.

If $j \in S$, because $i \vDash j$, and $i \notin S$, we have $S \backslash\{j\} \in \pi$. But $k \in S \backslash\{j\}$, and $j \vDash k$, thus $S \backslash\{j, k\} \in \pi$, and using monotonicity, $S \backslash\{k\} \in \pi$.

To prove that the internal domination relation is transitive, assume that $i \unrhd j \unrhd k$, and that $i \neq j \neq k \neq i$. Suppose that $S \in \pi$ is such that $i, k \in S$. We want to prove that $S \backslash\{k\} \in \pi$. There are two possibilities:

If $j \in S$, because $j \unrhd k$, we have $S \backslash\{k\} \in \pi$.

If $j \notin S$, let $S^{\prime}=S \cup\{j\}$. Because $S^{\prime} \in \pi$ (due to the monotonicity of $\phi$ ), $j, k \in S^{\prime}$, and $j \unrhd k$, we have $S^{\prime} \backslash\{k\}=$ $(S \cup\{j\}) \backslash\{k\} \in \pi$. But $i, j \in S^{\prime} \backslash\{k\}$, and $i \unrhd j$ implies $S^{\prime} \backslash\{j, k\}=S \backslash\{k\} \in \pi$, as desired.

To prove that the criticality relation is transitive, assume that $i \succeq j \succeq k$, and that $i \neq j \neq k \neq i$. Suppose that $S \subseteq N \backslash\{i, k\}$ is such that $S \cup\{k\} \in \pi$, and we want to prove that $S \cup\{i\} \in \pi$. There are two possibilities:

If $j \notin S$, then we get $S \subseteq N \backslash\{j, k\}$; and because $j \succeq k$, and $S \cup\{k\} \in \pi$, we have $S \cup\{j\} \in \pi$. But it is also true that $S \subseteq N \backslash\{i, j\}$; and because $i \succeq j$, we have $S \cup\{i\} \in \pi$.

If $j \in S$, let $S^{\prime}=S \backslash\{j\}$. Then $S^{\prime} \cup\{k\} \subseteq N \backslash\{i, j\}$, and $S^{\prime} \cup\{k\} \cup\{j\}=S \cup\{k\} \in \pi$. Because $i \succeq j$, we get $S^{\prime} \cup\{k\} \cup\{i\} \in \pi$. But $S^{\prime} \cup\{i\} \subseteq N \backslash\{j, k\}$; and because $j \succeq k$, and $S^{\prime} \cup\{k\} \cup\{i\} \in \pi$, we get $S^{\prime} \cup\{i\} \cup\{j\}=S \cup\{i\} \in \pi$. $\square$

\section{B. Proof of Proposition 2.3}

To see (i), assume that $i \vDash j, S \in \pi^{m}$, and $j \in S$. Then, if $i \notin S$, it would be $S \backslash\{j\} \in \pi$, but this contradicts the minimal character of the path set $\pi$. Conversely, assuming the hypothesis, let $T \in \pi$ be such that $j \in T$, and $i \notin T$. We must prove that $T \backslash\{j\} \in \pi$. Let $S \in \pi^{m}$ be such that $S \subseteq T$. If $j \in S$ it would be $i \in S$, and thus $i \in T$ (contradiction). Thus $j \notin S$; and as a consequence, $S \subseteq T \backslash\{j\}$, and $T \backslash\{j\} \in \pi$.

To prove (ii) assume that $i \unrhd j, S \in \pi^{m}$, and $j \in S$. Then $T=S \cup\{i\} \in \pi$, and $i, j \in T$; and thus $T \backslash\{j\}=(S \cup$ $\{i\}) \backslash\{j\} \in \pi$. Conversely, assume the hypothesis, and let $T \in$ $\pi$ be such that $i, j \in T$. We must prove that $T \backslash\{j\} \in \pi$. Let $S \in$ $\pi^{m}$ be such that $S \subseteq T$. Now, if $j \in S$ then $(S \cup\{i\}) \backslash\{j\} \subseteq$ $(T \cup\{i\}) \backslash\{j\}=T \backslash\{j\}$, and thus $T \backslash\{j\} \in \pi$. On the other hand, if $j \notin S$, then $S \subseteq T \backslash\{j\}$, and thus $T \backslash\{j\} \in \pi$.

Finally, to prove (iii), assume that $i \succeq j$, and let $S \in \pi^{m}$ be such that $j \in S$, and $i \notin S$. Because $S=(S \backslash\{j\}) \cup\{j\} \in \pi$, we get $(S \backslash\{j\}) \cup\{i\}=(S \cup\{i\}) \backslash\{j\} \in \pi$. Conversely, assume the hypothesis, and consider $S \subseteq N \backslash\{i, j\}$ such that $S \cup\{j\} \in$ $\pi$. We want to prove that $S \cup\{i\} \in \pi$. Let $T \in \pi^{m}$ be such that $T \subseteq S \cup\{j\}$. Now, if $j \in T$, we get $(T \cup\{i\}) \backslash\{j\} \in \pi$, and thus $S \cup\{i\} \in \pi$. If $j \notin T$, then $T \subseteq S$, and so $S \cup\{i\} \in \pi$. $\square$ 


\section{Proof of Proposition 2.6}

To prove (i), notice that if $i \vDash j$, because there is no $S \in \pi^{m}$ such that $j \in S$, and $i \notin S$, then we get $i \succeq j$.

If $i \vdash j$, because $j \not \models i$, there exists $S \in \pi^{m}$ such that $i \in S$, but $j \notin S$. Now, $T=(S \cup\{j\}) \backslash\{i\} \notin \pi$, because if $T \in \pi$, it would be $T \backslash\{j\}=S \backslash\{i\} \in \pi$ (because $i \vDash j$ ), which is a contradiction. Thus, $j \nsucceq i$, and this proves (ii).

It is clear from Proposition 2.3 that $i \unrhd j$ implies $i \succeq j$. If $i \triangleright j$, because $j \not i$, there exist $S \in \pi^{m}$ such that $i \in S$, but $(S \cup\{j\}) \backslash\{i\} \notin \pi$. Now $j \notin S$, because if $j \in S$, it would be $S \backslash\{j\} \in \pi$ (because $i \unrhd j$ ), which is a contradiction. Thus, $j \nsucceq i$.

\section{Proof of Proposition 2.7}

If $i \neq j$, then using Definition 2.1, we have the relations $i \vDash j$, and $i \unrhd j$ iff $j \in S \in \pi$ implies $S \backslash\{j\} \in \pi$, and this property characterizes irrelevant components.

\section{E. Proof of Proposition 3.1}

If $i \neq j$, we can write

$$
\begin{aligned}
& h\left(1_{i}, 1_{j}, \mathbf{p}\right)=\sum_{\substack{S \in \pi \\
i, j \in S}} \prod_{\substack{k \in S \\
k \neq i, j}} p_{k} \prod_{k \notin S}\left(1-p_{k}\right) . \\
& h\left(0_{i}, 0_{j}, \mathbf{p}\right)=\sum_{\substack{S \in \pi \\
i, j \notin S}} \prod_{k \in S} p_{k} \prod_{\substack{k \notin S \\
k \neq i, j}}\left(1-p_{k}\right) . \\
& h\left(1_{i}, 0_{j}, \mathbf{p}\right)=\sum_{\substack{S \in \pi \\
i \in S, j \notin S}} \prod_{\substack{k \in S \\
k \neq I}} p_{k} \prod_{\substack{k \notin S \\
k \neq j}}\left(1-p_{k}\right) \\
& h\left(0_{i}, 1_{j}, \mathbf{p}\right)=\sum_{\substack{S \in \pi \\
i \notin S, j \in S}} \prod_{\substack{k \in S \\
k \neq j}} p_{k} \prod_{\substack{k \notin S \\
k \neq i}}\left(1-p_{k}\right)
\end{aligned}
$$

(i) If $i \vDash j$, every addend in (4) appears also in (2) so that $h\left(0_{i}, 0_{j}, \mathbf{p}\right)-h\left(0_{i}, 1_{j}, \mathbf{p}\right) \geq 0$. Using Lemma 1.1-(iv), $h\left(0_{i}, 0_{j}, \mathbf{p}\right) \leq h\left(0_{i}, 1_{j}, \mathbf{p}\right)$, and thus $h\left(0_{i}, 0_{j}, \mathbf{p}\right)=h\left(0_{i}, 1_{j}, \mathbf{p}\right)$. For the second part, if $i \not \models j$, then there is at least one addend in (4) which is not in (2). If $\mathbf{p} \in(0,1)^{n}$, this addend is strictly positive, which implies $h\left(0_{i}, 0_{j}, \mathbf{p}\right)<h\left(0_{i}, 1_{j}, \mathbf{p}\right)$.

(ii) If $i \unrhd j$, every addend in (1) appears also in (3), and thus $h\left(1_{i}, 0_{j}, \mathbf{p}\right)-h\left(1_{i}, 1_{j}, \mathbf{p}\right) \geq 0$. Using Lemma 1.1-(iv), $h\left(1_{i}, 0_{j}, \mathbf{p}\right) \leq h\left(1_{i}, 1_{j}, \mathbf{p}\right)$, and thus $h\left(1_{i}, 0_{j}, \mathbf{p}\right)=h\left(1_{i}, 1_{j}, \mathbf{p}\right)$. For the second part if $i \not j$, there is at least one addend in (1) which is not in (3). If $\mathbf{p} \in(0,1)^{n}$, this addend is strictly positive, which implies $h\left(1_{i}, 0_{j}, \mathbf{p}\right)<h\left(1_{i}, 1_{j}, \mathbf{p}\right)$.

(iii) If $S \in \pi$ is such that $i \notin S$, and $j \in S$, then $S=$ $(S \backslash\{j\}) \cup\{j\} \in \pi$, and $S \backslash\{j\} \subseteq N \backslash\{i, j\}$. Thus, if $i \succeq$ $j$, then $(S \cup\{i\}) \backslash\{j\} \in \pi$, and so every addend in (4) appears also in (3). This implies $h\left(1_{i}, 0_{j}, \mathbf{p}\right)-h\left(0_{i}, 1_{j}, \mathbf{p}\right) \geq$ 0.

(iv) Finally, if $i \succeq j$, but $j \nsucceq i$, there exist $T \subseteq N \backslash\{i, j\}$ such that $S=T \cup\{i\} \in \pi$, and $T \cup\{j\} \notin \pi$. This means that there is at least one addend in (3) which does not appear in (4). If $\mathbf{p} \in(0,1)^{n}$, this addend is not zero, and we get the desired result.

\section{F. Proof of Proposition 3.2}

If $i \not \models j$, there exists $S \in \pi$ such that $j \in S, i \notin S$, and $S \backslash\{j\} \notin \pi$. Because $i \succeq j$, and $S=(S \backslash\{j\}) \cup\{j\}$, then we get $(S \backslash\{j\}) \cup\{i\} \in \pi$. Define $\mathbf{p} \in[0,1]^{n}$ by

$$
p_{k}=\left\{\begin{array}{lll}
1 & \text { if } \quad k \in S \backslash\{j\} \\
x & \text { if } \quad k=i \\
y & \text { if } \quad k=j \\
0 & \text { otherwise }
\end{array}\right.
$$

Then, using Lemma 1.1-(v), $I_{i}^{B}(\mathbf{p})-I_{j}^{B}(\mathbf{p})=x-y$, because from (1)-(4), $h\left(1_{i}, 1_{j}, \mathbf{p}\right)=h\left(1_{i}, 0_{j}, \mathbf{p}\right)=h\left(0_{i}, 1_{j}, \mathbf{p}\right)=1$, and $h\left(0_{i}, 0_{j}, \mathbf{p}\right)=0$; so (i) is proved.

To prove (ii), assume that $i \not j$. Then there exists $S \in \pi$ such that $i, j \in S$, and $S \backslash\{j\} \notin \pi$. Because $i \succeq j$, we get $S \backslash\{i\} \notin \pi$. Define $\mathbf{p} \in[0,1]^{n}$ by

$$
p_{k}= \begin{cases}1 & \text { if } \quad k \in S \backslash\{i, j\} \\ x & \text { if } \quad k=i \\ y & \text { if } \quad k=j \\ 0 & \text { otherwise }\end{cases}
$$

Then using Lemma 1.1-(v), $I_{i}^{B}(\mathbf{p})-I_{j}^{B}(\mathbf{p})=y-x$, because from (1)-(4), then $h\left(1_{i}, 1_{j}, \mathbf{p}\right)=1$, and $h\left(1_{i}, 0_{j}, \mathbf{p}\right)=$ $h\left(0_{i}, 1_{j}, \mathbf{p}\right)=h\left(0_{i}, 0_{j}, \mathbf{p}\right)=0$.

Finally, if $i \nsucceq j$, there exists $S \subseteq N \backslash\{i, j\}$ such that $S \cup$ $\{j\} \in \pi$, and $S \cup\{i\} \notin \pi$. Define $\mathbf{p} \in[0,1]^{n}$ by

$$
p_{k}=\left\{\begin{array}{llr}
1 & \text { if } & k \in S \\
x & \text { if } & k=I \\
y & \text { if } & k=j \\
0 & \text { otherwise }
\end{array}\right.
$$

Then using Lemma 1.1-(v), $I_{i}^{B}(\mathbf{p})-I_{j}^{B}(\mathbf{p})=-1$, because from (1)-(4), then $h\left(1_{i}, 1_{j}, \mathbf{p}\right)=h\left(0_{i}, 1_{j}, \mathbf{p}\right)=1$, and $h\left(1_{i}, 0_{j}, \mathbf{p}\right)=h\left(0_{i}, 0_{j}, \mathbf{p}\right)=0$, so (iii) is proved.

\section{G. Proof of Theorem 3.3}

If $i \vDash j$, then using Lemma 1.1-(v), and Proposition 3.1, we get

$$
\begin{aligned}
I_{i}^{B}(\mathbf{p})-I_{j}^{B}(\mathbf{p})=\left(p_{j}-p_{i}\right)[ & \left.h\left(1_{i}, 1_{j}, \mathbf{p}\right)-h\left(1_{i}, 0_{j}, \mathbf{p}\right)\right] \\
& +\left[h\left(1_{i}, 0_{j}, \mathbf{p}\right)-h\left(0_{i}, 1_{j}, \mathbf{p}\right)\right] .
\end{aligned}
$$

But, because $i \vDash j$, from Proposition 2.6, we get $i \succeq j$, and from Proposition 3.1 , we get $h\left(1_{i}, 0_{j}, \mathbf{p}\right)-h\left(0_{i}, 1_{j}, \mathbf{p}\right) \geq 0$. Now, taking into account Lemma 1.1-(iv), if $p_{i} \leq p_{j}$, then $I_{i}^{B}(\mathbf{p})-$ $I_{j}^{B}(\mathbf{p}) \geq 0$. This proves (i).

Because $j$ is relevant, and $i \neq j$, if $i \vDash j$, it must be $i \not 1$; and if $\mathbf{p} \in(0,1)^{n}$, from Proposition 3.1(ii), we get $h\left(1_{i}, 1_{j}, \mathbf{p}\right)>$ $h\left(1_{i}, 0_{j}, \mathbf{p}\right)$. Proceeding as in the proof of part (i), we have $I_{i}^{B}(\mathbf{p})>I_{j}^{B}(\mathbf{p})$.

To prove (iii), assume that $i \vdash j$, and $p_{i}>p_{j}$. It must be $i \not j$; and using Proposition 2.6, we get $i \succeq j$. From Proposition 3.2, there exists a reliability vector $\mathbf{p}^{*} \in[0,1]^{n}$ with $p_{i}^{*}=$ $p_{i}$, and $p_{j}^{*}=p_{j}$, such that $I_{i}^{B}\left(\mathbf{p}^{*}\right)-I_{j}^{B}\left(\mathbf{p}^{*}\right)=p_{j}-p_{i}<0$. On the other hand, because $i \vdash j$, using Proposition 2.6, we get $j \nsucceq i$. Thus, using Proposition 3.2, there exists $\mathbf{p}^{\diamond} \in[0,1]^{n}$ such that $p_{i}^{\diamond}=p_{i}, p_{j}^{\diamond}=p_{j}$, and $I_{i}^{B}\left(\mathbf{p}^{\diamond}\right)-I_{j}^{B}\left(\mathbf{p}^{\diamond}\right)=1>0$. 


\section{H. Proof of Theorem 3.4}

If $i \unrhd j$, then using Lemma 1.1-(v), and Proposition 3.1, we get

$$
\begin{aligned}
I_{i}^{B}(\mathbf{p})-I_{j}^{B}(\mathbf{p})=\left(p_{j}-p_{i}\right)[ & \left.h\left(0_{i}, 0_{j}, \mathbf{p}\right)-h\left(0_{i}, 1_{j}, \mathbf{p}\right)\right] \\
+ & {\left[h\left(1_{i}, 0_{j}, \mathbf{p}\right)-h\left(0_{i}, 1_{j}, \mathbf{p}\right)\right] . }
\end{aligned}
$$

But, because $i \unrhd j$, from Proposition 2.6, we get $i \succeq j$; and, from Proposition 3.1-(iii), $h\left(1_{i}, 0_{j}, \mathbf{p}\right)-h\left(0_{i}, 1_{j}, \mathbf{p}\right) \geq 0$. Now, taking into account Lemma 1.1-(v), if $p_{i} \geq p_{j}$, then $I_{i}^{B}(\mathbf{p})-$ $I_{j}^{B}(\mathbf{p}) \geq 0$. This proves (i).

Because $j$ is relevant, $i \neq j$, and $i \unrhd j$, then using Proposition 2.7 , we get $i \not \nvdash j$. Now, because $\mathbf{p} \in(0,1)^{n}$, then using the converse implication of Proposition 3.1-(i), we have $h\left(0_{i}, 1_{j}, \mathbf{p}\right) \neq h\left(0_{i}, 0_{j}, \mathbf{p}\right)$. But, due to the second part of Lemma 1.1-(iv), this is equivalent to $h\left(0_{i}, 1_{j}, \mathbf{p}\right)<h\left(0_{i}, 0_{j}, \mathbf{p}\right)$. Finally, proceeding as in the proof of part (i) of this theorem, and taking into account that $p_{i}>p_{j}$, we get $I_{i}^{B}(\mathbf{p})>I_{j}^{B}(\mathbf{p})$.

To prove part (iii), assume that $i \triangleright j, j$ is relevant, and $p_{i}<$ $p_{j}$. Using Proposition 2.6, it must be that $i \not k j$, and $i \succeq$ $j$; and, from Proposition 3.2, there exists a reliability vector $\mathbf{p}^{*} \in[0,1]^{n}$ with $p_{i}^{*}=p_{i}$, and $p_{j}^{*}=p_{j}$, such that $I_{i}^{B}\left(\mathbf{p}^{*}\right)-$ $I_{j}^{B}\left(\mathbf{p}^{*}\right)=p_{i}-p_{j}<0$. On the other hand, because $i \triangleright j$, using Proposition 2.6, we get $j \nsucceq i$. Thus, using Proposition 3.2, there exists $\mathbf{p}^{\diamond} \in[0,1]^{n}$ such that $p_{i}^{\diamond}=p_{i}, p_{j}^{\diamond}=p_{j}$, and $I_{i}^{B}\left(\mathbf{p}^{\diamond}\right)-I_{j}^{B}\left(\mathbf{p}^{\diamond}\right)=1>0$.

\section{Proof of Theorem 3.5}

If $p_{i}=p_{j}$, then using Lemma 1.1-(v), we get $I_{i}^{B}(\mathbf{p})-$ $I_{j}^{B}(\mathbf{p})=h\left(1_{i}, 0_{j}, \mathbf{p}\right)-h\left(0_{i}, 1_{j}, \mathbf{p}\right)$. But, if $i \succeq j$, from Proposition $3.1, h\left(1_{i}, 0_{j}, \mathbf{p}\right)-h\left(0_{i}, 1_{j}, \mathbf{p}\right) \geq 0$. This proves part (i).

Part (ii) is derived in the same way.

To prove part (iii), assume that $i \succ j$, and $p_{i} \neq p_{j}$. If $i \nvdash j$, because $i \succ j$, then we get also $i \not \models j$; and from Proposition 3.2, there exists $\hat{\mathbf{p}} \in[0,1]^{n}$ such that $\hat{p}_{i}=p_{i}, \hat{p}_{j}=p_{j}$, and $I_{i}^{B}(\hat{\mathbf{p}})-$ $I_{j}^{B}(\hat{\mathbf{p}})=p_{i}-p_{j}$. If $i \not j$, because $i \succ j$, then we get also $i \not j$; and from Proposition 3.2, there exists $\tilde{\mathbf{p}} \in[0,1]^{n}$ such that $\tilde{p}_{i}=p_{i}, \tilde{p}_{j}=p_{j}$, and $I_{i}^{B}(\tilde{\mathbf{p}})-I_{j}^{B}(\tilde{\mathbf{p}})=p_{j}-p_{i}$. Thus, if $i \nvdash j$, and $i \not j$, we can always select $\mathbf{p}^{*}$ such that $I_{i}^{B}\left(\mathbf{p}^{*}\right)<$ $I_{j}^{B}\left(\mathbf{p}^{*}\right)$. On the other hand, because $j \nsucceq i$, using Proposition 3.2 there exists $\mathbf{p}^{\diamond} \in[0,1]^{n}$ such that $p_{i}^{\diamond}=p_{i}, p_{j}^{\diamond}=p_{j}$, and $I_{i}^{B}\left(\mathbf{p}^{\diamond}\right)-I_{j}^{B}\left(\mathbf{p}^{\diamond}\right)=1>0$.

\section{ACKNOWLEDGMENT}

The authors thank the referees for their carefully reading of an earlier version of this paper, and for pointing out a number of useful comments and references.

\section{REFERENCES}

[1] Z. Birnbaum, "On the importance of different components in a multicomponent system," in Multivariate Analysis. New York, USA: Academic Press, 1969, vol. 2.

[2] P. Boland, F. Proschan, and Y. Tong, "Optimal arrangements of components via pairwise rearrangements,," Naval Research Logistics, vol. 36, pp. 807-815, 1989.

[3] D. Butler, "A complete importance ranking for components of binary coherent systems with extensions to multi-state systems," Naval Research Logistics Quarterly, vol. 4, 1979.

[4] F. Hwang, "A hierarchy of importance indices," IEEE Trans. Reliability, vol. 54, no. 1, pp. 169-172, 2005.

[5] F. Meng, "Some further results on ranking the importance of system components," Reliability Engineering and System Safety, vol. 47, no. 2, pp. 97-101, 1995.

[6] J. Mi, "A unified way of comparing the reliability of coherent systems," IEEE Trans. Reliability, vol. 52, no. 1, pp. 38-43, 2003.

[7] F. Meng, "Comparing Birnbaum importance measure of system components," Probability in the Engineering and Informational Sciences, vol. 18, pp. 237-245, 2004.

[8] F. Meng, "Comparing the importance of system components by some structural characteristics," IEEE Trans. Reliability, vol. 45, no. 1, pp. $59-65,1996$.

[9] F. Meng, "Relationships of Fussell-Vesely and Birnbaum importance to structural importance in coherent systems," Reliability Engineering and System Safety, vol. 67, pp. 55-60, 2000.

[10] P. Boland and E. El-Neweihi, "Measures of component importance in reliability theory," Computers Ops Res, vol. 4, pp. 455-463, 1995.

[11] M. Rausand and A. Høyland, System reliability theory: Models, statistical methods and applications, 2nd ed. Hoboken, New Jersey, USA: John Wiley \& Sons, 2004.

[12] P. Boland and F. Proschan, "The reliability of k out of n systems," Ann. Probability, vol. 11, pp. 760-764, 1983.

[13] F. Lin and W. Kuo, "Reliability importance and invariant optimal allocation," Journal of Heuristics, vol. 8, pp. 155-171, 2002.

[14] W. Kuo, V. Prasad, F. Tillman, and C. Hwang, Optimal Reliability Design: Fundamentals and Applications. Cambridge, UK: Cambridge University Press, 2001.

[15] W. Kuo and V. Prasad, "An annotated overview of system reliability optimization," IEEE Trans. Reliability, vol. 49, pp. 176-187, 2000.

[16] J. Isbell, "A class of majority games," Quart. J. Math. Oxford Ser., vol. 7, no. 2, pp. 183-187, 1956.

[17] A. Taylor and W. Zwicker, Simple games: Desirability relations, trading, and pseudoweightings. , New Jersey, USA: Princeton University Press, 1999.

[18] F. Carreras and J. Freixas, "Complete simple games," Mathematical Social Sciences, vol. 32, pp. 139-155, 1996.

Josep Freixas received the $\mathrm{PhD}$ in Mathematics from the Technical University of Catalonia, in 1994. He has worked in the Department of Mathematics 3 at Manresa. He was a visiting professor in the Bergamo University (Italy) in 1996, and in the Union College of Schenectady in New York (USA) in 2000. His research interest includes Decision and game theory, reliability and statistics, and computer sciences. He has published many papers on these topics.

Montserrat Pons received the $\mathrm{PhD}$ in Mathematics from the University of Barcelona in 1984 with a thesis on uniform structures on partially ordered sets. Her research interests include ordered sets and lattice theory, fuzzy sets, game theory, and systems control and reliability. She has published papers in all of these areas. 